Research Paper

\title{
Iron Chelators and Exogenic Photosensitizers. Synergy through Oxidative Stress Gene Expression
}

\author{
Anna Mrozek-Wilczkiewicz ${ }^{1,2} \bowtie$, Katarzyna Malarz ${ }^{2,3}$, Marzena Rams-Baron ${ }^{1,2}$, Maciej Serda ${ }^{3}$, Daniela \\ Bauer ${ }^{4}$, Franz-Peter Montforts ${ }^{4}$, Alicja Ratuszna ${ }^{1,2}$, Thomas Burley ${ }^{5}$, Jaroslaw Polanski ${ }^{3}$ and Robert \\ Musiol $^{3 凶}$ \\ 1. A. Chełkowski Institute of Physics, University of Silesia in Katowice, Poland \\ 2. Silesian Center for Education and Interdisciplinary Research, University of Silesia in Katowice, Chorzów, Poland \\ 3. Institute of Chemistry, University of Silesia in Katowice, Poland \\ 4. Institute of Organic and Analytical Chemistry, University of Bremen, Germany \\ 5. The Institute of Cancer Research, London, United Kingdom \\ $\bowtie$ Corresponding author: anna.mrozek-wilczkiewicz@us.edu.pl or robert.musiol@us.edu.pl \\ (C) Ivyspring International Publisher. This is an open access article distributed under the terms of the Creative Commons Attribution (CC BY-NC) license \\ (https://creativecommons.org/licenses/by-nc/4.0/). See http://ivyspring.com/terms for full terms and conditions.
}

Received: 2016.10.17; Accepted: 2017.03.22; Published: 2017.07.05

\begin{abstract}
In non-invasive anticancer photodynamic therapy (PDT), a nontoxic photosensitizer (PS), which is activated by visible light, is used as a magic bullet that selectively destroys cancer cells. Recently, we described the combined therapy of 5 -aminolevulinic acid (ALA-PDT) with thiosemicarbazone (TSC), i.e. an iron-chelating agent. This resulted in a strong synergistic effect. Herein, we investigated a novel strategy using a combination of PDT consist of the xenobiotic-porphyrin type PS with TSC. We observed a synergistic effect for all of the pairs of TSC-PS. This approach can be rationalized by the fact that both chlorin and TSC can affect the generation of reactive oxygen species (ROS). In order to elucidate the plausible mechanism of action, we also combined the investigated PSs with DFO, which forms complexes that are redox inactive. We detected a slight antagonism or additivity for this combination. This may suggest that the ability of an iron chelator (IC) to participate in the production of ROS and the generation of oxidative stress is important.
\end{abstract}

Key words: photodynamic therapy, thiosemicarbazone, photosensitizer, chlorin derivative, combination therapy, oxidative stress

\section{Introduction}

Thiosemicarbazones are a new class of compounds that have been suggested for anticancer therapy. These compounds bind metal ions and form active complexes. Their mechanism of action is multitargeted and consists of the deprivation of iron from cytosol, the generation of reactive oxygen species and the inhibition of the activity of ribonucleotide reductase [1]. Iron is a microelement that participates in a variety of important processes such as electron transport, DNA synthesis, oxygen delivery and erythropoiesis. One consequence of iron binding is the inhibition of the cell-division cycle and the arrest of cells at the G1/S interphase [2]. Depletion of iron also has an influence on the regulation of important genes (NDRG1 and BNIP3), which are crucial for triggering the apoptosis of cancer cells [3]. This multitargeted mechanism of action makes this type of compounds especially attractive material for the study of their possible application in anticancer therapy. The most popular derivative of this class of compounds is Triapine (3-AP) (Figure 1), which is a promising candidate for an anticancer drug.

Currently, 3-AP is the first TSC ribonucleotide reductase inhibitor that has passed phase II of clinical trials [4]. Intensive research on the structure and activity of TSC has led to novel, highly active derivatives such as Dp44mT, which has been widely described in the literature $[5,6]$. More recently, TSC that are based on the 8-hydroxyquinoline moiety have been described by our group [7]. Among them, the 
compound MS168, which is a strong "booster" of protoporphyrin IX (PpIX), has been used in aminolevulinic acid-triggered photodynamic therapy [8]. Good results prompted us to try combination therapy with xenobiotic photosensitizers.

Photodynamic therapy is a method of cancer treatment that is characterized by a high selectivity relative to normal cells. Both the specific structure of PS and the organization of the tumor tissue together contribute to the selectivity of PDT. The essence of this therapy is the production of singlet oxygen and free radicals in the reaction of PS with light of a suitable wavelength [9]. This energy changes the trigger chain reactions in the cell, thus causing various types of damage and finally the destruction of the tumor. The most commonly used PS are derivatives from the group of porphyrins, chlorins, phthalocyanines, naphthalocyanines or texaphyrins [10]. In comparison to porphyrins, chlorins have the maximum of fluorescence shifted towards longer wavelengths, which makes them more useful in therapy. For our research, we chose a recently published arabinose-based polyol derivative of chlorin (chlorin c), which has superior photophysical properties [11,12]. Moreover, the amphiphilic nature of this molecule guarantees good penetration through the cellular membrane and distribution via blood circulation. Foscan, a chlorin that is a drug approved for the PDT of the squamous cell carcinoma in Europe [13], was another PS that was tested in our study.

Combination therapy is defined as the simultaneous use of more than one drug in order to achieve a therapeutic effect. When the total effect of a drug combination is greater than the sum of the
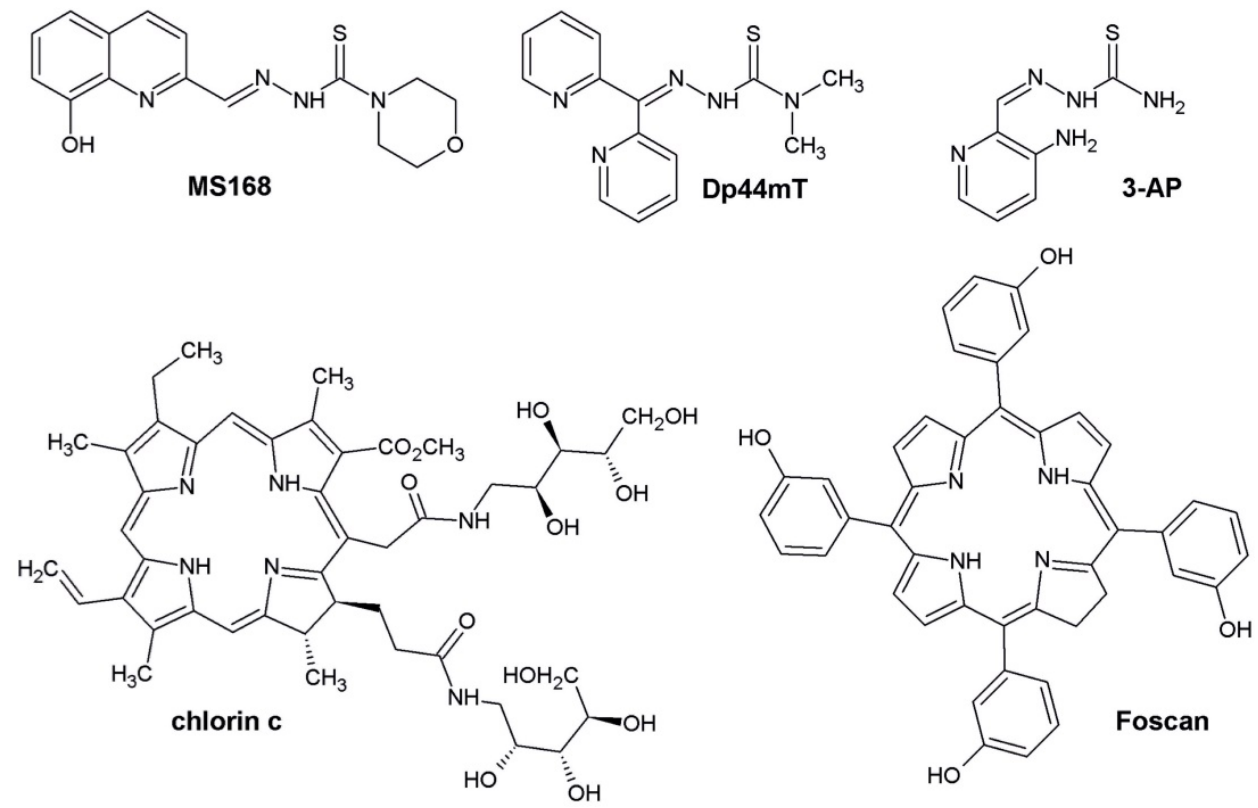

Fig. 1. Structures of TSCs (MS168, Dp44mT, 3-AP) and PSs (chlorin c, Foscan).

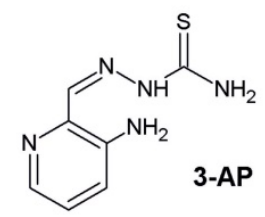

effects that are triggered by the drugs individually, the interaction is synergistic [14]. However, the increase of activity is not the only benefit from the combination therapy of active substances. Dose reduction while maintaining effectiveness, the reduction of toxicity and an attack on several molecular targets simultaneously are some examples of the gains.

As was mentioned above, we have recently shown that ALA-PDT can be efficiently boosted by the co-application of some redox-active TSCs, which may be explained by an increase in the formation of ROS. However, the mechanism of selective PpIX accumulation in a tumor is still not well understood. For example, the differences in iron metabolism and the expression of mitoferrins between a tumor and normal cells have recently been carefully investigated by Ogura et al. Those researchers found that the amount of mitochondrial labile iron ion and expression levels of mitochondrial iron transporters (mitoferrins) were lower in cancer cells than in normal cells [15]. Moreover, the metabolism of iron to heme is substantially lower in cancer cells. Consequently, the addition of sodium ferrous citrate as a source of iron resulted in lowering the accumulation of PpIX in both tumor and normal cells, but this level in cancer cells was still high enough to induce a therapeutic effect. This so-called Ogura effect is a good explanation for the remarkable selectivity of ALA-PDT. However, in the case of xenobiotic PS, the reverse effect was observed. Namely, the addition of iron during PDT treatment with phthalocyanines [16], chlorins $[17,18]$ or porfimer [19] increased the effectivity of the therapy. This phenomenon may be connected with an increased ability to form ROS during an induced Fenton reaction. The addition of iron chelators such as DFO diminished cell death in PDT because DFO complexes with iron do not have redox potency $[20,21]$. In this regard, the interactions between iron chelators and exogenic PSs are particularly interesting. It is commonly known that the generation of ROS influences the cellular redox environment and leads to the induction of oxidative stress. There are many protective mechanisms in cells that destabilize radicals. The 
most common are the genes that are responsible for the production of the proteins superoxide dismutase (MnSOD) and catalase (CAT). These two molecules are responsible for the degradation of superoxide and hydrogen peroxide, respectively. These genes are part of the natural defense of a cell, i.e. their antioxidant potential as well as their important, but still not clear role in diseases, have been reported [22]. Changes in the expression of such protective genes are a typical response in therapy with cytostatics or PDT $[23,24]$. Gołąb et al. showed that PDT induced the overexpression of MnSOD in cancer cells [25]. In colorectal cancer cells (HCT116) that are investigated in current study, the expression of MnSOD is strongly connected with activity of p53 [26]. The upregulation of MnSOD results in higher level of $\mathrm{H}_{2} \mathrm{O}_{2}$ generated in mitochondria and subsequent increase its concentration in cytosol, where it plays important role as signaling molecule. However this role is not yet fully understood, it has been proven to participate in cells proliferation activity in several cancers [27]. The vague function of MnSOD and $\mathrm{H}_{2} \mathrm{O}_{2}$ in cell is well illustrated by the changing classification of the dismutase encoding gene as tumor suppressor or oncogene [22]. In this regard the role of CAT as peroxide discharging protein become even more important. At lease in several cancers including prostate and colon cancer the ratio of MnSOD/CAT appeared to be crucial for cancer spread or treatment prognosis [28]. Moreover the catalase overexpression seems to induce better treatment sensitivity in cancer cells [29].

Table 1. Calculated combination indexes for the combination of TSC and PS.

\begin{tabular}{lll}
\hline CI[a] & chlorin c & Foscan \\
\hline MS168 & $0.82 \pm 0.17$ & $0.60 \pm 0.10$ \\
Dp44mT & $0.51 \pm 0.10$ & $0.26 \pm 0.14$ \\
3 -AP & $0.59 \pm 0.13$ & $0.35 \pm 0.11$ \\
DFO & $1.13 \pm 0.20$ & $1.05 \pm 0.19$ \\
\hline
\end{tabular}

[a]for $\mathrm{Fa}=0.9, \mathrm{CI}>1$ - antagonism, $\mathrm{CI}=1$ - additivity, $\mathrm{CI}<1$ - synergy. Experiments were performed on the HCT116 cell line (see Supporting Information).

Thus in our study we decided to investigate also the overall antioxidant response of the cell to combination therapy. For the similar mechanism of action of PDT and TSC drugs this may allow to clear out the intracellular mechanism laying beneath effectivity of phototherapy.

\section{Results and Discussion}

Among the factors that influence drug-drug interactions, the duration of an experiment and the concentrations applied play important, but unclear roles [14]. Thus, we decided to measure the cytotoxic effect after the simultaneous application of PS and
TSC. An incubation of $48 \mathrm{~h}$ was selected as the optimal duration of the experiment. The cytotoxic effects were measured by determining the fraction of living cells by means of MTS assay. Concentrations of the tested compounds were selected based on $\mathrm{IC}_{50}$ (see SI Table S2). The interaction type and strength was assumed through a combination index (CI), which was calculated according to the useful method that was proposed by Chou [30]. As is summarized in Table 1, all of the CIs take a value $<1$ for combinations with MS168, Dp44mT and 3-AP and >1 for DFO.

Accordingly, we observed synergy for all of the combinations except DFO. The strongest synergy was observed for the combination of Dp44mT and Foscan $(\mathrm{CI}=0.26)$, while the result for the combination of MS168 and chlorin c showed a considerably weaker interaction ( $\mathrm{CI}=1$ for the simple additivity). For DFO, we observed additivity/antagonism in combination with both of the PSs that were investigated. These results are particularly interesting as the tested PSs are exogenic compounds therefore, their concentration is independent of the iron level. Consequently, the mechanism of the interactions is different from the PpIX boosting that was demonstrated for ALA-PDT combined with an IC. We can hypothesize that both drugs interact complementarily via mechanisms that are based on oxidative stress. As was discussed in the introduction, TSCs generate reactive oxygen species in a Fenton reaction, thus leading to cellular damage [31]. At the same time, the absorption of energy by PSs results in a photodynamic reaction with oxygen, thereby producing more reactive singlet oxygen forms $\left({ }^{1} \mathrm{O}_{2}\right)$. Therefore, both PS and TSC produce ROS. It is well known that free radical reactions are very quick and cause damage that affects only a limited cellular area. Thus, the drug localization in a cell plays a crucial role for the efficiency of PDT [18,32]. As was highlighted above, for the phthalocyanine photosensitizer PC-4, the mitochondrial level of iron affected the overall effectivity of PDT in human head and neck carcinoma cells (UMSCC1) [16]. Therefore, in our experiments, we first verified the localization of the drugs that were being investigated. After incubation with the tested TSCs and PSs, the cells were stained with dyes that bind to mitochondria. The results of the experiments using a confocal microscope are shown in Figure 2. Analysis of the images indicated that all of the drugs were localized in mitochondria. Therefore, the observed co-localization may be crucial for the mutual action in combination therapy where TSC may interfere with the labile mitochondrial iron metabolism through an iron-depleting action or may have other more complex effects through a specific accumulation. 
The mechanism of cytotoxicity in PDT and TSC treatment may rely on the formation of ROS. With this in mind, the observed co-localization in mitochondria is a desirable feature. Singlet oxygen generation is considered to be the main factor that is responsible for cellular damage in PDT. Iron chelators may at least indirectly participate in the formation of ROS. On the other hand, DFO, due to its hexadentate complex with $\mathrm{Fe}(\mathrm{III})$, is unable to participate in a Fenton reaction and in the generation of ROS [33,34].

Moreover, at high concentrations, DFO is known
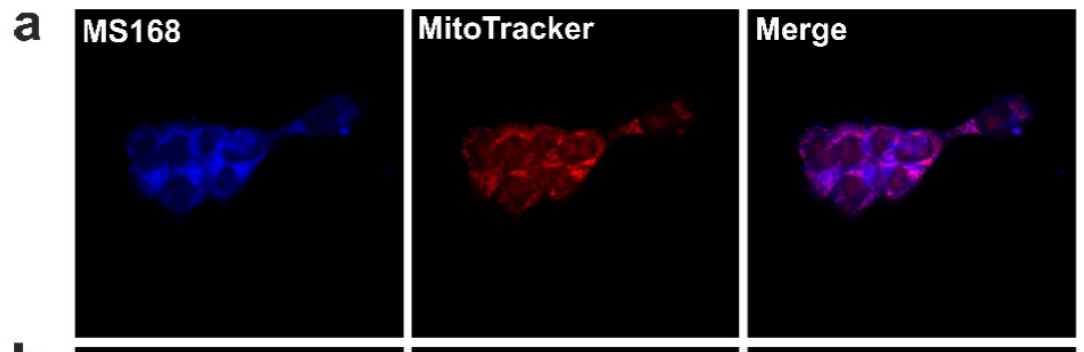

b Dp44mT
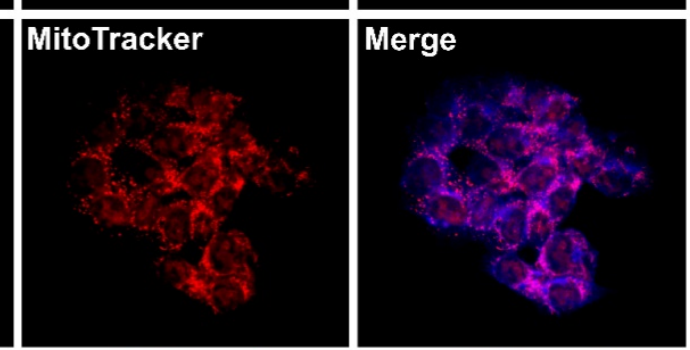

C 3-AP
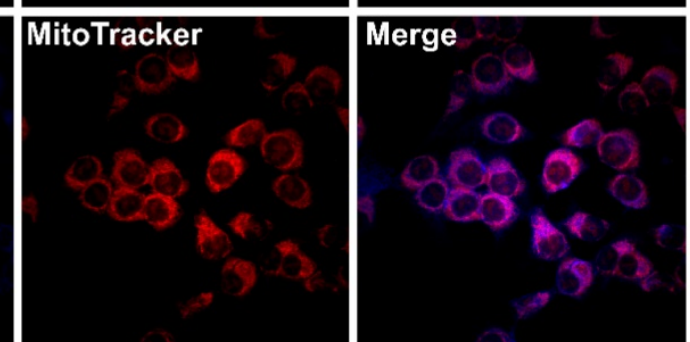

d
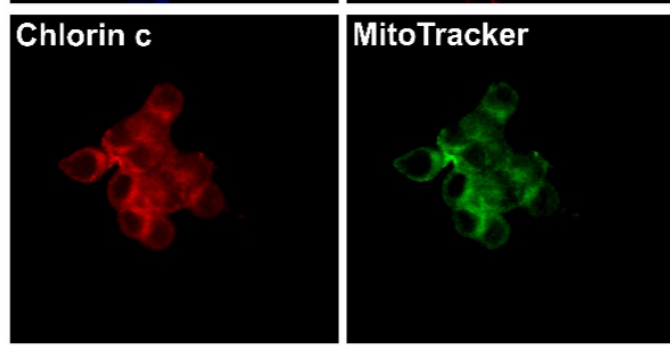

e
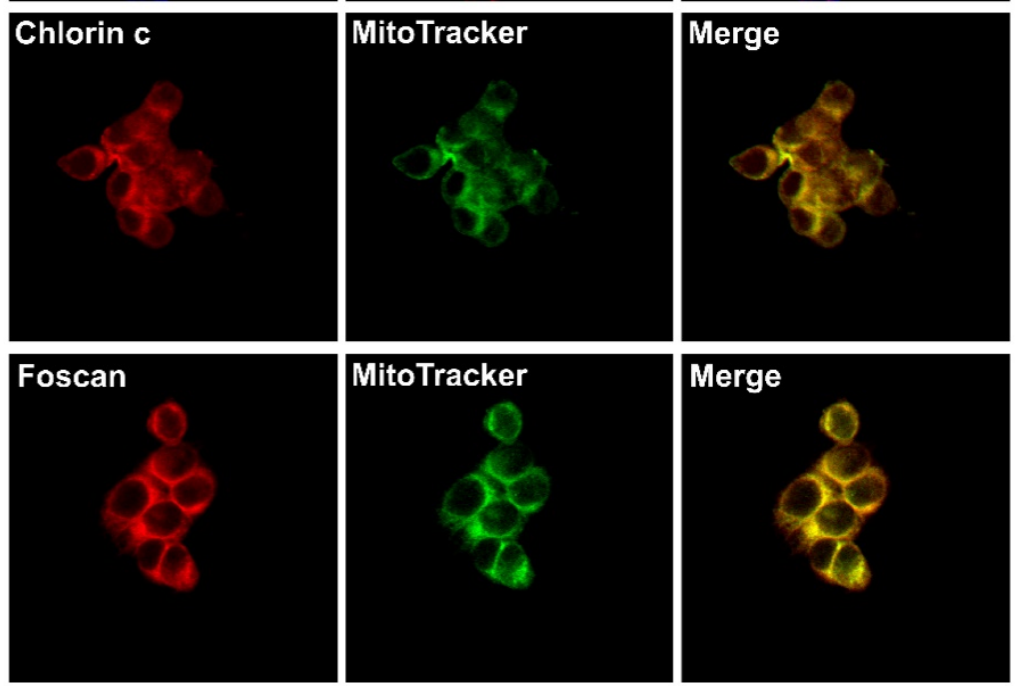

Fig. 2. Cellular localization of TSCs; MS168 (a), Dp44mT (b), 3-AP (c) and PSs; chlorin c (d), Foscan (e) in the HCT1 16 cell line. First panel - fluorescence of the investigated compounds alone, middle panel - colocalization with mitochondria marker (MitoTracker $®$ Orange for TSC's and Green for PS's), last panel - merge. Scale bar represents $50 \mu \mathrm{m}$. for its anti-oxidant potency [35]. This feature is in agreement with our results, which are shown in Table 1. To evaluate this hypothesis, we designed an experiment to measure the singlet oxygen yield after combination therapy in DMEM solutions. Our results confirmed that the only PS is responsible for ${ }^{1} \mathrm{O}_{2}$ generation during PDT-TSC treatment (Figure A). Solutions of either MS168 or Dp44mT alone did not generate ${ }^{1} \mathrm{O}_{2}$ after irradiation. In contrast, irradiated PS solutions and mixtures of PS-TSC revealed enhanced fluorescence, which clearly indicated ${ }^{1} \mathrm{O}_{2}$ generation. In this regard, the additional ROS effects of TSC may, through different species, influence the overall homeostasis of the cell.

The possible effects of ROS generation may be different types of cellular damage including lipid peroxidation. In further experiments, we checked the influence of the investigated combinations (TSC+PS) on the oxidation processes in a cell (Figure B). At all of the concentrations, the signals of the combination therapy were stronger than those of chelators. In general, some differences between the two PSs - chlorin c and Foscan - were observed. For chlorin c, which is a less potent PS, a stronger malondialdehyde (MDA) signal was observed in all of the combinations with both TSCs. Foscan, however, has a stronger cytotoxic effect (compare Table S1 in Supporting Information). In this case, its simultaneous application with TSCs resulted in a stronger lipid peroxidation only at lower concentrations. High concentrations gave a comparable or even lower signal of MDA in the Foscan-TSCs due to the overkill effect.

ROS-degrading proteins are other important elements of the total antioxidative potential of the cells. As was already mentioned, the level of MnSOD and CAT may be increased after chemotherapy. This is a natural mechanism of defense against elevated oxidative damage in cells. The MnSOD in mitochondria can dismutate the $\mathrm{O}_{2}{ }^{\bullet}$ - radical into $\mathrm{H}_{2} \mathrm{O}_{2}$. Then the hydrogen peroxide level is regulated by CAT in cytosol. During the induced oxidative stress, those 

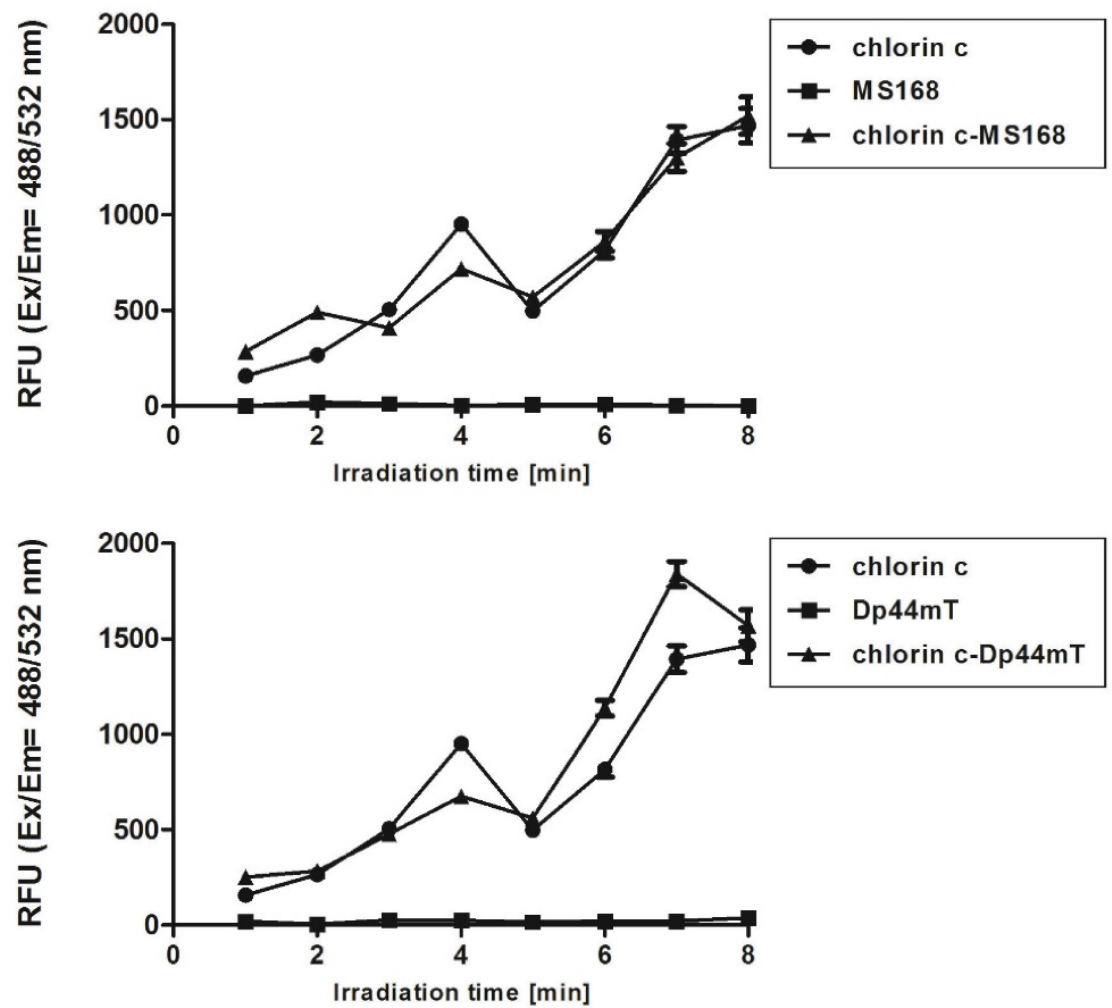

Figure A . Fluorescence response of the Singlet Oxygen Sensor Green reagent to the ${ }^{\prime} \mathrm{O}_{2}$ that was generated during the irradiation of DMEM solutions containing the investigated PSs and TSCs as well as their combinations. The duration of the irradiation that was applied corresponds to a light dose $4-32 \mathrm{~J} / \mathrm{cm}^{2}$. breaking down the antioxidative potential of the cell. Iron chelators acting through Haber-Weiss and Fenton reactions stimulate the cell machinery to reduce the elevated ROS concentration. Similar effect is observed in PS although in smaller extent. Increased production of oxygen radicals in mitochondria results in correspondingly higher concentration of $\mathrm{H}_{2} \mathrm{O}_{2}$, which causes upregulation of CAT to maintain the equilibrium. However higher level of $\mathrm{H}_{2} \mathrm{O}_{2}$ may also facilitate the Fenton reaction generating more ROS and leading to the cell death. Thus lowering the CAT expression may increase effectivity of TSC therapy [36]. On the other hand higher CAT activity produce more oxygen which may enhance the PDT process as recently reported by Min et al. [37]. The exact mechanism of CAT downregulation is still not well understood, however it can be hypothesized that interaction with vital metal cations may play a role as reported elsewhere [38]. enzymes and particularly catalase potency may affect the cell survival. The addition of 2-methoxyestradiol, which is an MnSOD inhibitor, has been reported to improve PDT [25]. In this regard, we hypothesized that influencing the overall antioxidative potential of the cell may change its vulnerability to therapy. To verify this assumption we estimated the expression of MnSOD and CAT in response to the investigated therapies. As is shown in Figure $\mathrm{C}$, the addition of a neocuproine (NCP) ROS scavenger and a chelating agent resulted in lowering the expression of genes in cancer cells. Foscan increased the expression of both genes while chlorin c amplified the level of CAT but the MnSOD to a lesser degree. Both chelators strongly increased the gene levels, although in a different manner. Dp44mT affected the expression of CAT to a higher degree than MnSOD. The overall expression of genes in the Dp44mT-treated cells was also considerably higher. This was in agreement with the combination indexes that suggested a stronger synergy for this thiosemicarbazone. In all cases, the level of ROS-degrading proteins decreased with the addition of neocuproine. Noticeably, the combination of Dp44mT with Foscan (the strongest synergy) caused a lower expression of CAT than any of those drugs alone. This effect was also confirmed with the addition of NCP. Thus the combination of two drugs inducing the redox damage in the cell may results in

\section{Conclusions}

In conclusion, we have shown that the co-application of the tested TSCs in xenobiotic-chlorin mediated PDT can exert a synergistic effect that is apparently due to its engagement in the labile mitochondrial iron metabolism and the formation of ROS. The synergistic effect is limited to only redox-active chelators, as the mobilization does not change the PS concentration. Thiosemicarbazones accumulate in mitochondria and by redox-active complexes generate ROS, whose are substrates for MnSOD/CAT enzymes. Combination therapy lead to decrease overall cell antioxidative potency by downregulation of CAT. The strong interaction between Dp44mT and Foscan can be explained on this basis. The investigated strategy can be a valuable option for increasing the efficiency of PDTs, e.g. for therapies that use Foscan, which is an approved PDT drug. As described above there is strong interest in enhanced photodynamic therapy by addition of adjuvant drugs. According to the mechanistic consideration the chelation of iron seems to be effective way. However the literature reports do not fully support such hypothesis. With this in mind our results help to understand the baseline mechanism beneath photodynamic - adjuvant interactions. 
Photodynamic therapy still growing and is intensively investigated. Until now different PSs are available as endogenic PpIX and chlorin derivatives. Iron chelators are another extensively growing group of anticancer agents with two drugs currently under the clinical trials 3-AP and DpC analog of Dp44mT (NCT02688101). With this in mind presented results may have particular importance for design clinical experiments as different drugs require specific approach to obtain positive results.

\section{Experimental section}

\section{Cell lines}

The human colon cancer cell line HCT116 was obtained from ATCC. The cells were grown as monolayer cultures in Dulbecco's Modified Eagle's Medium (DMEM) supplemented with 12\% (HCT116) Fetal Bovine Serum (Sigma) and standard antibiotics in $75 \mathrm{~cm}^{2}$ flasks (Nunc). Cells were cultured under standard conditions at $37^{\circ} \mathrm{C}$ in a humidified atmosphere at $5 \% \mathrm{CO}_{2}$ and $37^{\circ} \mathrm{C}$ and were passaged every 3-4 days as required.

\section{Cellular localization}

The cells were seeded onto glass slides at a
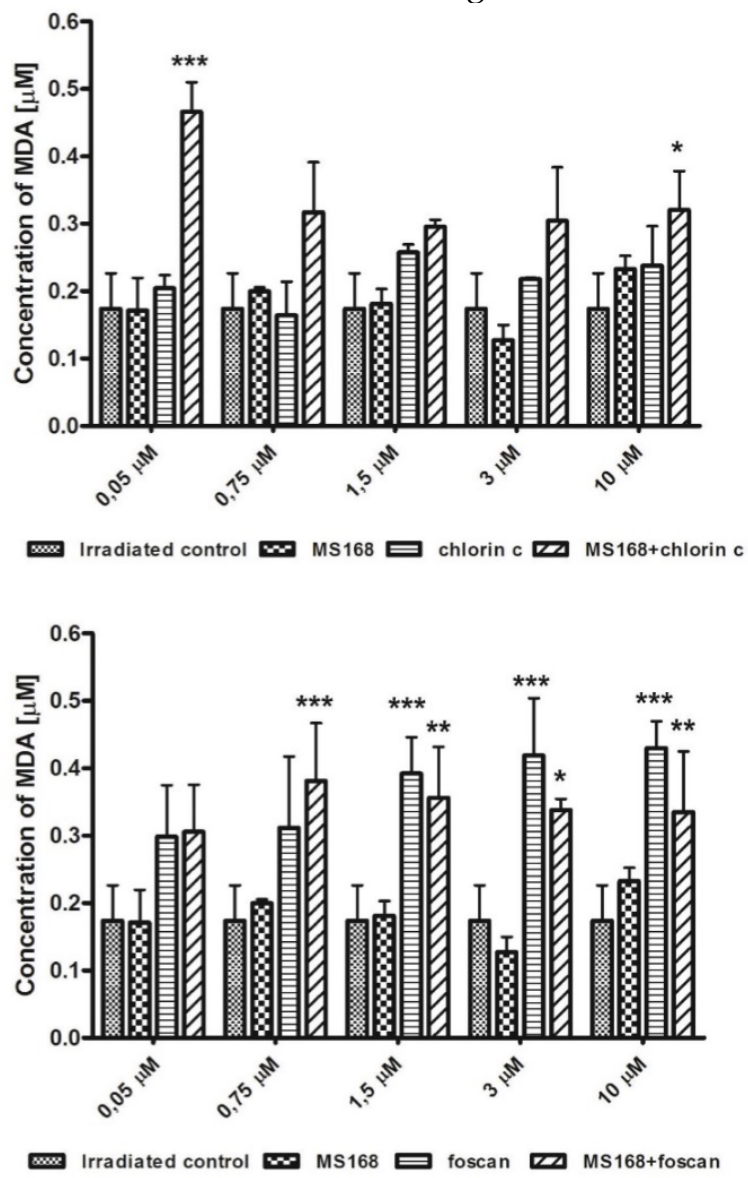

density of $3 \cdot 10^{5}$ cells/slide and incubated at $37^{\circ} \mathrm{C}$ for $24 \mathrm{~h}$. Then, the medium was removed and solutions of Dp44mT (15 nM), MS168 (15 nM), 3-AP (1.67 $\mu \mathrm{M})$ and PS $(2.5 \mu \mathrm{M})$ were added. The next day, the cells washed with PBS after which MitoTracker ${ }^{\circledR}$ Orange (100 nM, $30 \mathrm{~min}$ incubation, Molecular Probes) or MitoTracker ${ }^{\circledR}$ Green (100 nM, 30 min incubation, Molecular Probes) were added. After staining with organelle markers, the cells were washed with PBS and fixed by $3.7 \%$ paraformaldehyde for 10 minutes. Intracellular localization was observed using an Olympus Fluoview FV1000 confocal laser scanning system equipped with an Olympus IX81 inverted microscope (Olympus, Poland). TSCs were excited using the laser $405 \mathrm{~nm}$ line, MitoTracker Green was excited using the argon-ion laser $488 \mathrm{~nm}$ line, photosensitizers and MitoTracker Orange by the helium-neon laser $543 \mathrm{~nm}$ line. The fluorescence of TSCs, MitoTracker Green, PS and MitoTracker Orange were monitored using an emission filters: 461; 519; 615 and $576 \mathrm{~nm}$, respectively. Image acquisition was performed using a $60 x$ oil immersion objective lens. The analysis and processing of images were performed using an ImageJ 1.41 (Wayne Rasband, National Institutes of Health, USA).
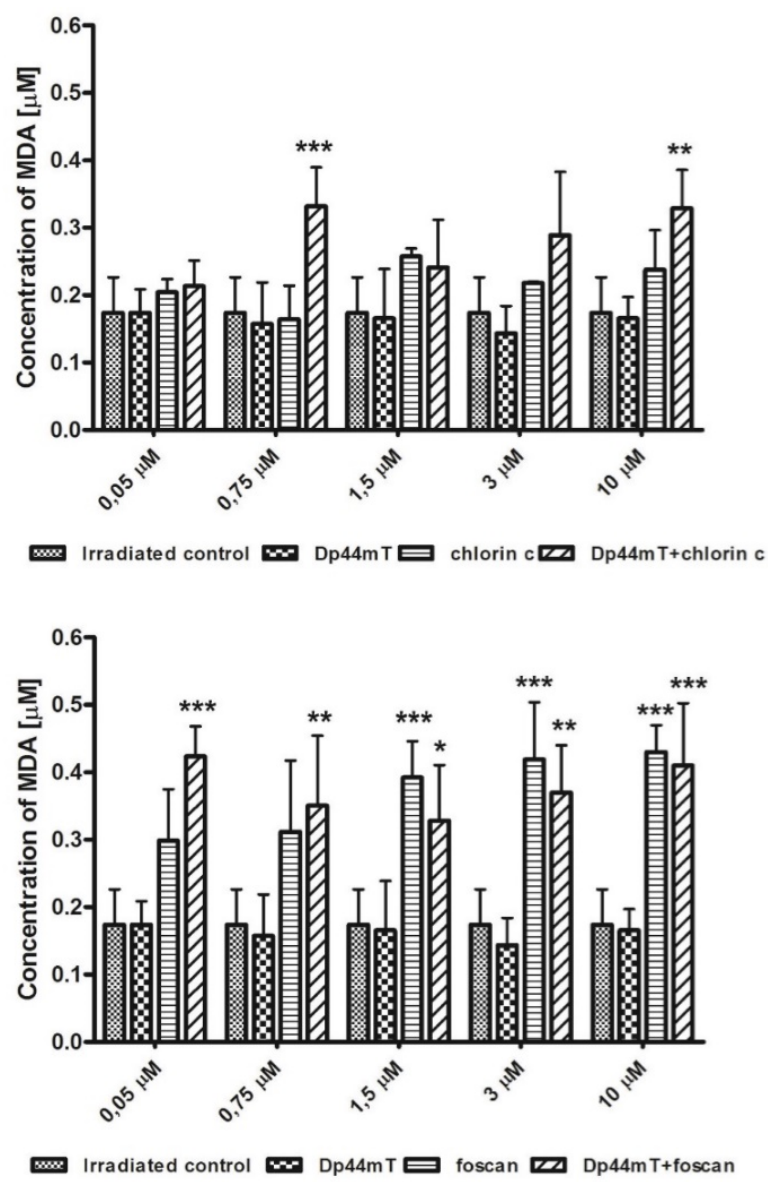

Figure B. Lipid peroxidation of the HCT116 cell line after incubation with TSCs, PSs and their combinations. Results are shown as the mean \pm SD of three independent measurements. Data were analyzed using one-way ANOVA with Bonferroni's post-hoc test: ${ }^{2} p<0.05$, $*^{*} p<0.01, * * * p<0.001$ as compared to the irradiated control (untreated) group. 

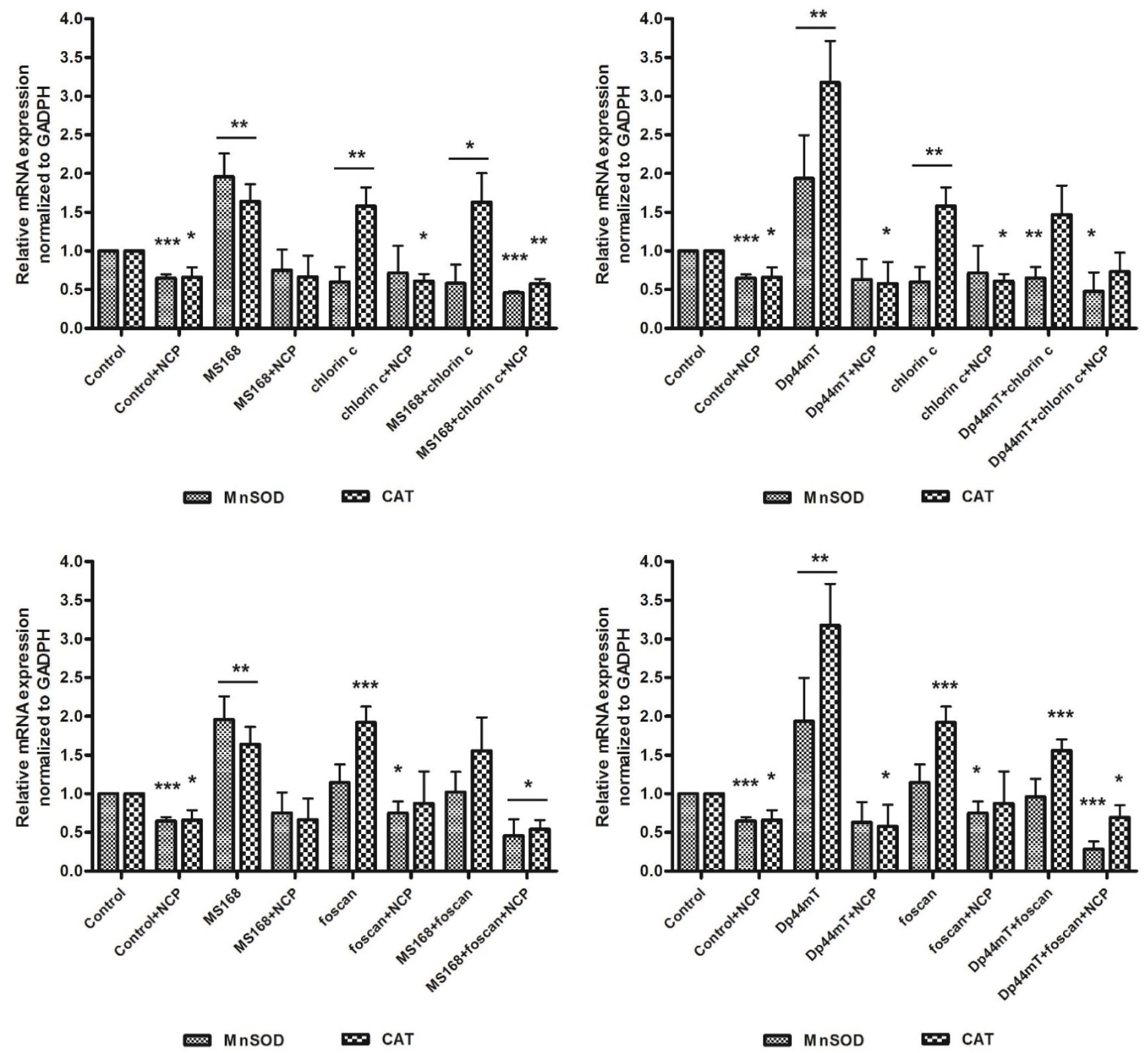

Figure C. mRNA expression of MnSOD and CAT in the HCT1 16 cell line after incubation with TSCs, PSs and their combinations. Results are shown as the mean \pm SD of three independent measurements. Data were analyzed using the Student's t-test: ${ }^{p} p<0.05, * * 0.01, * * * p<0.001$ as compared to the irradiated control (untreated) group.

\section{Generation of singlet oxygen}

In order to detect singlet oxygen $\left({ }^{1} \mathrm{O}_{2}\right)$ in irradiated solutions that contained various combinations of the investigated compounds, we used Singlet Oxygen Sensor Green (SOSG) (Invitrogen). After reaction with ${ }^{1} \mathrm{O}_{2}$, this selective reagent produces fluorescent SOSG endoperoxides that after excitation at $\lambda=488 \mathrm{~nm}$ emit a strong green fluorescence with a maximum at $\lambda=532 \mathrm{~nm}$. The stock solution of the investigated compounds was diluted in DMEM to a final concentration of $10 \mu \mathrm{M}$. Irradiation was performed in a black 96-well plate using an LED light source (Thorlabs, $\lambda=660 \mathrm{~nm}$ ) for various time intervals. In order to a homogeneous illumination of the samples only four wells were irradiated in each cycle. The stock solution (in $\mathrm{MeOH}$ ) was added to each well in a final concentration of 10 $\mu \mathrm{M}$ prior to the SOSG. The fluorescent spectra were measured using spectrofluorimeter Hitachi F-7000 before and after each irradiation period.

\section{Lipid peroxidation}

The levels of the lipid peroxidation products were measured using a modified method of Thiobarbituric Acid Reactive Substances (TBARS). The cells were seeded in $3 \mathrm{~cm}$ Petri dishes (Nunc) at a density of $3 \cdot 10^{5}$ cells/dish and incubated at $37^{\circ} \mathrm{C}$. After $24 \mathrm{~h}$, solutions of TSCs and PSs were prepared at concentrations of $0.05,0.75,1.5,3,10 \mu \mathrm{M}$ and were 
added. The next day, the solution was removed and the cells were washed with PBS after which $1 \mathrm{~mL}$ of DMEM (without phenol red and without FBS) was added. The cells were irradiated with a light dose 12 $\mathrm{J} / \mathrm{cm}^{2}$ at wavelength of $660 \mathrm{~nm}$ and then incubated at $37^{\circ} \mathrm{C}$. After $3 \mathrm{~h}$, the cells were washed with PBS and trypsinized with $0.05 \%$ Trypsin-EDTA solution (Sigma), then centrifuged for $2 \mathrm{~min}$ at 14,000 rpm. The cells were suspended in PBS, and then $15 \%$ trichloroacetic acid (Sigma) in $0.25 \mathrm{M} \mathrm{HCl}$ and $0.37 \%$ thiobarbituric acid (Sigma) in $0.25 \mathrm{M} \mathrm{HCl}$ were added. All of the samples were incubated at $90^{\circ} \mathrm{C}$ for 20 minutes, then quick chilled on ice for 5 minutes and centrifuged for $5 \mathrm{~min}$ at $5,000 \mathrm{rpm}$. The absorbance of supernatant was measured using a multi-plate reader (Synergy 4, Bio Tek) at $532 \mathrm{~nm}$. The concentration of the final product of lipid peroxidation, malondialdehyde (MDA) was calculated using a molar extinction coefficient of $156 \mathrm{mM}^{-1} \mathrm{~cm}^{-1}$. The experiments were performed three times.

\section{Oxidative stress mRNA expression of MnSOD and CAT}

The cells were seeded in $3 \mathrm{~cm}$ Petri dishes (Nunc) at a density $0.5 \cdot 10^{6}$ cells and incubated overnight. Then, the medium was removed and solutions of Dp44mT (25 nM), MS168 $(25 \mathrm{nM})$ and PS $(0.5 \mu \mathrm{M})$ were added. For quenching ROS, Neocuproine (NCP) at a concentration of $25 \mu \mathrm{M}$ was applied. The next day, the cells were irradiated with a light dose of 12 $\mathrm{J} / \mathrm{cm}^{2}$ at a wavelength of $660 \mathrm{~nm}$ and then incubated at $37^{\circ} \mathrm{C}$. After $6 \mathrm{~h}$, total RNA was isolated from the cells using TRIzol Reagent (Ambion) according to the manufacturer's instructions. cDNA synthesis was performed with $5 \mu \mathrm{g}$ of total RNA using a GoScript TM Reverse Transcriptase kit (Promega) and Oligo(dT) 23 Primers (Sigma). The relative mRNA levels of the MnSOD and CAT genes in the TSC-PS-PDT-treated cells were determined in comparison with the levels of the untreated controls without PDT. Real-Time PCR was performed using a CTX96 Touch $^{\mathrm{TM}}$ Real-Time PCR Detection System (Biorad) at a reaction volume of $20 \mu \mathrm{L}$. The reaction consisted of SYBR Green Mix (Biorad), primers pair mix $(0.5 \mu \mathrm{M}$ each) and $1 \mu \mathrm{L}$ cDNA. All of the primer pair sequences were purchased from Sigma-Aldrich and are listed in Table S2. The experiment was set up under the following conditions: initial denaturation at $95^{\circ} \mathrm{C}$ for $20 \mathrm{sec}$; followed by 40 denaturation cycles at $95^{\circ} \mathrm{C}, 10 \mathrm{sec}$; annealing (primer-specific temperature for $20 \mathrm{sec}$ ) and extension at $72^{\circ} \mathrm{C}$ for $30 \mathrm{sec}$. Data was analyzed based on a comparison of the expression of the target genes to a reference GADPH gene using the of $2^{-\triangle \Delta C T}$ method.

\section{Statistical analysis}

All of the data are presented as mean values and standard deviations (SD). Statistical differences in the expression of the genes were calculated using the Student's t-test. Statistical analysis of lipid peroxidation was performed using one-way ANOVA with a Bonferroni post-hoc test (comparisons of multiple groups against the control). A p-value of 0.05 or less was considered to be statistically significant. GraphPad Prism v.5.0 software (GraphPad Software, USA) was used for statistical. CI indexes with error values were calculated in the CompuSyn software.

\section{Supplementary Material}

Supplementary tables.

http://www.jcancer.org/v08p1979s1.pdf

\section{Acknowledgments}

The financial support of the National Science Centre grants 2014/13/D/NZ7/00322 (A.M.W.), and 2013/09/B/NZ7/00423 (R.M.) is greatly appreciated.

\section{Competing Interests} interest.

The authors declare no competing financial

\section{References}

1. Kalinowski DS, Yu Y, Sharpe PC, Islam M, Liao Y-T, Lovejoy DB, et al. Design, synthesis, and characterization of novel iron chelators: structure-activity relationships of the 2-benzoylpyridine thiosemicarbazone series and their 3-nitrobenzoyl analogues as potent antitumor agents. J Med Chem. 2007;50: 3716-29. doi:10.1021/jm070445z

2. Richardson DR, Milnes K. The potential of iron chelators of the pyridoxal isonicotinoyl hydrazone class as effective antiproliferative agents II: the mechanism of action of ligands derived from salicylaldehyde benzoyl hydrazone and 2-hydroxy-1-naphthylaldehyde benzoyl hydrazone. Blood. 1997;89: 3025-38.

3. Whitnall M, Howard J, Ponka P, Richardson DR. A class of iron chelators with a wide spectrum of potent antitumor activity that overcomes resistance to chemotherapeutics. Proc Natl Acad Sci U S A. 2006;103: 14901-6. doi:10.1073/pnas.0604979103

4. Mackenzie MJ, Saltman D, Hirte H, Low J, Johnson C, Pond G, et al. A Phase II study of 3-aminopyridine-2-carboxaldehyde thiosemicarbazone (3-AP) and gemcitabine in advanced pancreatic carcinoma. A trial of the Princess Margaret hospital Phase II consortium. Invest New Drugs. 2007;25: 553-8. doi:10.1007/s10637-007-9066-3

5. Jansson PJ, Yamagishi T, Arvind A, Seebacher N, Gutierrez E, Stacy A, et al. Di-2-pyridylketone 4,4-Dimethyl-3-thiosemicarbazone (Dp44mT) Overcomes Multidrug-Resistance by a Novel Mechanism Involving the Hijacking of Lysosomal P-Glycoprotein (Pgp). J Biol Chem. 2015; 290(15):9588-9603. doi:10.1074/jbc.M114.631283

6. Richardson DR, Sharpe PC, Lovejoy DB, Senaratne D, Kalinowski DS, Islam $\mathrm{M}$, et al. Dipyridyl thiosemicarbazone chelators with potent and selective antitumor activity form iron complexes with redox activity. J Med Chem. 2006; 49: 6510-21. doi:10.1021/jm0606342

7. Serda M, Kalinowski DS, Rasko N, Potůčková E, Mrozek-Wilczkiewicz A, Musiol R, et al. Exploring the Anti-Cancer Activity of Novel Thiosemicarbazones Generated through the Combination of Retro-Fragments: Dissection of Critical Structure-Activity Relationships. PLoS One. 2014; 9: e110291. doi:10.1371/journal.pone.0110291

8. Mrozek-Wilczkiewicz A, Serda M, Musiol R, Malecki G, Szurko A, Muchowicz A, et al. Iron Chelators in Photodynamic Therapy Revisited: Synergistic Effect by Novel Highly Active Thiosemicarbazones. ACS Med Chem Lett. 2014; 5: 336-339. doi:10.1021/ml400422a

9. MacCormack M. Photodynamic Therapy. Adv Dermatol. 2006; 22: 219-258. doi:10.1016/j.yadr.2006.09.008

10. Allison RR, Downie GH, Cuenca R, Hu X-H, Childs CJ, Sibata CH. Photosensitizers in clinical PDT. Photodiagn Photodyn Ther. 2004; 1: 27-42. doi:10.1016/S1572-1000(04)00007-9 
11. Bauer D, Montforts F-P. Chlorinderivate und deren Verwendung. DE 102010056443 A1, 2010.

12. Bauer D, Montforts F-P, Losi A, Görner H. Photoprocesses of chlorin e6 glucose derivatives. Photochem Photobiol Sci. 2012; 11: 925. doi:10.1039/c1pp05303e

13. Lorenz KJ, Maier H. Plattenepithelkarzinome im Kopf-Hals-Bereich. HNO. 2008; 56: 402-409. doi:10.1007/s00106-007-1573-1

14. Musiol R, Mrozek-Wilczkiewicz A, Polanski J. Synergy Against Fungal Pathogens: Working Together is Better Than Working Alone. Curr Med Chem. 2014; 21: 870-93.

15. Hayashi $M$, Fukuhara $H$, Inoue $K$, Shuin $T$, Hagiya $Y$, Nakajima $M$, et al. The Effect of Iron Ion on the Specificity of Photodynamic Therapy with 5-Aminolevulinic Acid. Missirlis F, editor. PLoS One. 2015; 10: e0122351. doi:10.1371/journal.pone.0122351

16. Hung H-I, Schwartz JM, Maldonado EN, Lemasters JJ, Nieminen A-L. Mitoferrin2-Dependent Mitochondrial Iron Uptake Sensitizes Human Head and Neck Squamous Carcinoma Cells to Photodynamic Therapy. J Biol Chem. 2013; 288: 677-86. doi:10.1074/jbc.M112.422667

17. Kessel D, Reiners JJ. Enhanced Efficacy of Photodynamic Therapy via a Sequential Targeting Protocol. Photochem Photobiol. 2014; 90(4):889-95. doi:10.1111/php.12270

18. Ali-Seyed M, Bhuvaneswari R, Soo KC, Olivo M. PhotolonTM Photosensitization induces apoptosis via ROS-mediated cross-talk between mitochondria and lysosomes. Int J Oncol. 2011; 39: 821-831. doi:10.3892/ijo.2011.1109

19. Kelley EE, Domann FE, Buettner GR, Oberley LW, Burns CP. Increased efficacy of in vitro Photofrin photosensitization of human oral squamous cell carcinoma by iron and ascorbate. J Photochem Photobiol B. 1997; 40: 273-7.

20. Zhang W, Wei H, Frei B. The iron chelator, desferrioxamine, reduces inflammation and atherosclerotic lesion development in experimental mice. Exp Biol Med. 2010; 235: 633-641. doi:10.1016/j.surg.2006.10.010.Use

21. Halliwell B. Protection against tissue damage in vivo by desferrioxamine: what is its mechanism of action? Free Radic Biol Med. 1989; 7: 645-51.

22. Dhar SK, St Clair DK. Manganese superoxide dismutase regulation and cancer. Free Radic Biol Med. 2012; 52: 2209-2222. doi:10.1016/j.freeradbiomed.2012.03.009

23. Saczko J, Kulbacka J, Chwilkowska A, Pola A, Lugowski M, Marcinkowska A, et al. Cytosolic superoxide dismutase activity after photodynamic therapy, intracellular distribution of Photofrin II and hypericin, and P-glycoprotein localization in human colon adenocarcinoma. Folia Histochem Cytobiol. 2007; 45: 93-97.

24. Piotrowska H, Kucinska M, Murias M. Expression of CYP1A1, CYP1B1 and MnSOD in a panel of human cancer cell lines. Mol Cell Biochem. 2013; 383: 95-102. doi:10.1007/s11010-013-1758-8

25. Gołab J, Nowis D, Skrzycki M, Czeczot H, Barańczyk-Kuźma A, Wilczyński GM, et al. Antitumor effects of photodynamic therapy are potentiated by 2-methoxyestradiol: A superoxide dismutase inhibitor. J Biol Chem. 2003; 278 : 407-414. doi:10.1074/jbc.M209125200

26. Behrend L, Mohr A, Dick T, Zwacka RM. Manganese Superoxide Dismutase Induces p53-Dependent Senescence in Colorectal Cancer Cells. Mol Cell Biol. 2005; 25: 7758-7769. doi:10.1128/MCB.25.17.7758-7769.2005

27. Lennicke C, Rahn J, Lichtenfels R, Wessjohann L a, Seliger B. Hydrogen peroxide - production, fate and role in redox signaling of tumor cells. Cell Commun Signal. Cell Communication and Signaling; 2015; 13: 39. doi:10.1186/s12964-015-0118-6

28. Miar A, Hevia D, Muñoz-Cimadevilla H, Astudillo A, Velasco J, Sainz RM, et al. Manganese superoxide dismutase (SOD2/MnSOD)/catalase and SOD2/GPx1 ratios as biomarkers for tumor progression and metastasis in prostate, colon, and lung cancer. Free Radic Biol Med. 2015; 85: 45-55. doi:10.1016/j.freeradbiomed.2015.04.001

29. Glorieux C, Dejeans N, Sid B, Beck R, Calderon PB, Verrax J. Catalase overexpression in mammary cancer cells leads to a less aggressive phenotype and an altered response to chemotherapy. Biochem Pharmacol. 2011; 82: 1384-1390. doi:10.1016/j.bcp.2011.06.007

30. Chou T. Theoretical Basis, Experimental Design, and Computerized Simulation of Synergism and Antagonism in Drug Combination Studies. Pharmacol Rev. 2007; 58: 621-681.

31. Yu Y, Rahmanto YS, Hawkins CL, Richardson DR. The Potent and Novel Thiosemicarbazone Chelators Di-2- Crucial Thiol Systems Required for Ribonucleotide Reductase. Mol Pharmacol. 2011 Jun;79(6):921-31.

32. Verhille M, Couleaud P, Vanderesse R, Brault D, Barberi-Heyob M, Frochot C. Modulation of photosensitization processes for an improved targeted photodynamic therapy. Curr Med Chem. 2010; 17: 3925-43.

33. Dean RT, Nicholson P. The action of nine chelators on iron-dependent radical damage. Free Radic Res. 1994; 20: 83-101.

34. Chaston TB, Richardson DR. Iron chelators for the treatment of iron overload disease: Relationship between structure, redox activity, and toxicity. Am J Hematol. 2003; 73: 200-210. doi:10.1002/ajh.10348

35. Link G, Konijn AM, Hershko C. Cardioprotective effect of alpha-tocopherol, ascorbate, deferoxamine, and deferiprone: mitochondrial function in cultured, iron-loaded heart cells. J Lab Clin Med. 1999; 133: 179-88.

36. Lee K-T, Lu Y-J, Mi F-L, Burnouf T, Wei Y-T, Chiu S-C, et al. Catalase-Modulated Heterogeneous Fenton Reaction for Selective Cancer Cell Eradication: $\mathrm{SnFe} 2 \mathrm{O} 4$ Nanocrystals as an Effective Reagent for Treating Lung Cancer Cells. ACS Appl Mater Interfaces. 2016; doi:10.1021/acsami.6b13529
37. Min JY, Lim S-O, Jung G. Downregulation of catalase by reactive oxygen species via hypermethylation of $\mathrm{CpG}$ island II on the catalase promoter. FEBS Lett. 2010; 584: 2427-32. doi:10.1016/j.febslet.2010.04.048

38. Patrushev N, Seidel-Rogol B, Salazar G. Angiotensin II requires zinc and downregulation of the zinc transporters $\mathrm{ZnT3}$ and $\mathrm{ZnT10}$ to induce senescence of vascular smooth muscle cells. PLoS One. 2012; 7: e33211. doi:10.1371/journal.pone.003321 